\title{
Effects of combined pharmacotherapy and psychotherapy for improving work functioning in major depressive disorder
}

Raymond W. Lam, Sagar V. Parikh, Rajamannar Ramasubbu, Erin E. Michalak, Edwin M. Tam, Auby Axler, Lakshmi N. Yatham, Sidney H. Kennedy and Chinnapalli V. Manjunath

\section{Background}

Major depressive disorder is associated with significant impairment in occupational functioning and reduced productivity, which represents a large part of the overall burden of depression.

\begin{abstract}
Aims
To examine symptom-based and work functioning outcomes with combined pharmacotherapy and psychotherapy treatment of major depressive disorder.

Method

Employed patients with a DSM-IV diagnosis of major depressive disorder were treated with escitalopram 10$20 \mathrm{mg} /$ day and randomised to: (a) telephone-administered cognitive-behavioural therapy (telephone CBT) $(n=48)$; or (b) adherence-reminder telephone calls $(n=51)$. Outcomes included the Montgomery-Åsberg Depression Rating scale (MADRS), administered by masked evaluators via telephone, and self-rated work functioning scales completed online. (Registered at clinicaltrials.gov: NCT00702598.)
\end{abstract}

\section{Results}

After 12 weeks, there were no significant between-group differences in change in MADRS score or in response/ remission rates. However, participants in the telephone-CBT group had significantly greater improvement on some measures of work functioning than the escitalopram-alone group.

\section{Conclusions}

Combined treatment with escitalopram and telephoneadministered CBT significantly improved some self-reported work functioning outcomes, but not symptom-based outcomes, compared with escitalopram alone.

\section{Declaration of interest}

R.W.L has received consulting and/or speaker honoraria from: AstraZeneca, Biovail, Eli Lilly, Lundbeck, Lundbeck Institute, Mochida, Pfizer, Servier, and Takeda; and has received research grants from: Aquaceutica, Bristol-Myers Squibb, Litebook Company, Lundbeck, Pfizer, St Jude Medical, and UBC Institute of Mental Health/Coast Capital Savings. S.V.P. has received consulting and/or speaker honoraria from: AstraZeneca, Bristol-Myers-Squibb, Eli Lilly, Lundbeck, and Pfizer; and has received research grants from: Apotex, AstraZeneca, Bristol-Myers-Squibb, Eli Lilly, Lundbeck, Novartis, Pfizer, and Servier; and holds shares in Mensante corporation. R.R. has received research grants from AstraZeneca. L.N.Y has received consulting and/or speaker honoraria from: Abbott, AstraZeneca, Bristol-Myers Squibb, Eli Lilly, Forest, GlaxoSmithKline, Johnson and Johnson, Novartis, Pfizer, Servier, and Wyeth; and has received research grants from: Janssen, Lundbeck, Otsuka, Ranbaxy. S.H.K. has received consulting and/or speaker honoraria from: Astrazeneca, Boehringer-Ingelheim, Bristol-Meyers Squibb, Brain Cells Inc, Eli Lilly, GlaxoSmithKline, Lundbeck, Pfizer, Servier, and St Jude Medical; and has received research grants from: AstraZeneca, Brain Cells Inc, Lundbeck, Pfizer, Servier, and St Jude Medical. E.E.M. has received consulting honoraria from Lundbeck.
As one of the most prevalent psychiatric conditions worldwide major depressive disorder is a leading cause of work-related disability and lost work productivity. ${ }^{1,2}$ The economic consequences of major depressive disorder represent a growing public health problem in many countries, and an escalating health concern globally. ${ }^{3-6}$ The costs of depression-related absenteeism (time off work) are high and increasing; for example, mental health issues (typically depression, anxiety and stress) contribute to $78 \%$ of short-term and $67 \%$ of long-term disability claims in Canada. ${ }^{7}$ However, compared with workers with other chronic medical conditions, people with major depressive disorder are more likely to continue working, even though depressive symptoms are associated with 'presenteeism' or decreased job performance and at-work productivity. ${ }^{8-11}$ The financial costs of presenteeism are much higher than those associated with absenteeism: Goetzel et $a l^{12}$ estimated the average productivity loss due to depressionrelated presenteeism as $15.3 \%$, compared with $10.7 \%$ loss due to absenteeism. In absolute dollars, the costs of depression-related productivity loss have been estimated as US $\$ 2$ billion monthly. ${ }^{13}$ Clearly, major depressive disorder has a significant negative impact on workers, their employers and the economy as a whole. ${ }^{14}$
Despite increasing recognition of the personal and economic burdens of major depressive disorder, outcomes for workers with depression remain poor and under-studied. ${ }^{15}$ A Cochrane review $^{16}$ identified only 11 randomised controlled trials (RCTs) on interventions aimed at reducing work disability in workers with major depressive disorder, with only 4 studies including work functioning as an outcome measure. The review did not find any convincing evidence that various interventions (including pharmacotherapy or psychosocial approaches) reduced workplace absence or improved occupational health outcomes in workers with depression. Therefore, significant gaps remain in our understanding of the most effective interventions for working people with major depressive disorder.

First-line recommended treatments for major depressive disorder include antidepressants and cognitive-behavioural therapy (CBT). ${ }^{17}$ Although CBT is well recognised as an effective intervention for depression, costs and time commitments may limit its availability and acceptability to workers needing treatment. However, newer methods of delivering CBT, including via telephone, allow for increased access at lower costs and greater convenience than traditional modes of psychological 
intervention. ${ }^{18}$ Initial studies in major depressive disorder have shown that telephone-delivered CBT is comparable to in-person $\mathrm{CBT}^{19}$ and leads to better outcomes than usual care alone. ${ }^{20,21}$ In fact, the evidence supporting telephone-based CBT is sufficient for it to be listed as a second-line treatment in some clinical guidelines. ${ }^{22}$ However, there are no RCTs specifically examining the combination of telephone-delivered psychotherapy with antidepressants. Combined treatment with in-person psychotherapy and pharmacotherapy has been associated with superior symptom outcomes in some studies, but not in others; systematic reviews and meta-analyses have shown that combination treatment had significantly superior efficacy, but with small effect sizes, compared with pharmacotherapy alone ${ }^{23}$ and psychotherapy alone. $^{24}$

Previous studies of combined treatment in depression have focused on symptom-based clinical outcomes. Importantly, there have been no studies examining combined pharmacotherapy and psychotherapy for improving work functioning in major depressive disorder. The main objectives of this proof of concept study were to test the hypotheses that, in employed patients with major depressive disorder of at least moderate severity, combining telephone-delivered CBT with an antidepressant would improve symptom and work productivity outcomes compared with an antidepressant alone.

\section{Method}

This trial received approval from the Institutional Research Boards at the University of British Columbia and each of the participating sites. Participants provided written informed consent before initiation of any study-related procedures. The study used a multicentre, single-blind (evaluator), randomised, parallel-groups design. All participants received open-label treatment with escitalopram $10-20 \mathrm{mg} /$ day for the entire 12 -week study period, and were randomised at baseline to: (a) the active condition, with eight weekly sessions of telephone-administered CBT (telephoneCBT group); or (b) the control condition, with eight weekly telephone adherence-reminder calls (escitalopram-alone group).

\section{Participants}

Participants were recruited from clinic referrals and by advertising at the three sites (Vancouver, Calgary and Toronto). Inclusion criteria were:

(a) male and female out-patients aged 19-65 years

(b) diagnosis of major depressive disorder by DSM-IV criteria, as confirmed using the Mini International Neuropsychiatric Interview $^{25}$

(c) current paid employment of $\geqslant 15 \mathrm{~h} /$ week

(d) score of 19 or higher on the Montgomery-Åsberg Depression Rating Scale ${ }^{26}$ (MADRS), indicating at least moderate depression, at both screening and baseline

(e) competency to give informed consent.

Participants were excluded for any of the following reasons:

(a) off work on short- or long-term disability

(b) pregnant or lactating women, and sexually active women of child-bearing potential who were not using medically accepted means of contraception

(c) serious suicidal risk as judged by the clinician

(d) unstable medical conditions

(e) diagnoses of organic mental disorders, substance misuse/ dependence, including alcohol, active within the past year; schizophrenia or other psychotic disorders; primary diagnosis of panic disorder, generalised anxiety disorder, obsessivecompulsive disorder, or post-traumatic stress disorder; bipolar disorder; eating disorders

(f) use of antidepressants or psychotropic drugs within 7 days of baseline visit (14 days for monoamine oxidase inhibitors, 5 weeks for fluoxetine)

(g) treatment-resistance in the current episode, as defined by failure (lack of clinically significant response) of two or more antidepressants at therapeutic doses for at least 6 weeks

(h) previous use of escitalopram or CBT for depression

(i) use of any additional treatment for depression during the study.

\section{Outcome assessments}

The main clinical outcome was change in the MADRS score from baseline to end-point. Other clinical outcomes included rates of response (defined as $\geqslant 50 \%$ improvement in MADRS scores to end-point) and remission (defined as end-point MADRS $\leqslant 12$ ). Planned key secondary work functioning outcomes were assessed using validated self-rated questionnaires, including: (a) the Work/ Role item from the Sheehan Disability Scale (SDS), ${ }^{27}$ rated on a 1-10 scale, with higher scores indicating greater impairment; (b) the Lam Employment Absence and Productivity Scale (LEAPS), ${ }^{28}$ consisting of a 7-item total score (range 0-28) and a 3-item productivity subscale (doing less work, doing poorer quality work, and making mistakes; range 0-12), with higher scores indicating greater difficulties at work; and (c) the Overall Performance item (range 0-10), with higher scores indicating better occupational performance), from the Health and Work Performance Questionnaire (HPQ), ${ }^{29}$ a comprehensive questionnaire assessing various aspects of work functioning. Other symptom, functional and quality of life measures were also administered for this study, but will be reported in subsequent papers.

\section{Procedures}

After a screening visit to establish eligibility and to obtain informed consent, participants were scheduled for five study visits (baseline, 2, 4, 8 and 12 weeks). At each study visit, psychiatrists monitored medications and documented adverse events. Within 2 days of each study visit, participants were rated using the MADRS over the telephone by trained independent evaluators, masked to treatment assignment and adverse events, using a structured interview guide. Interrater reliability of the evaluators was assessed using recorded interviews; the intraclass correlation was 0.93 for the MADRS. Participants also received an email with a link to a secure internet website to complete the study questionnaires.

At the baseline visit, participants were randomised 1:1 to one of the two study treatments (clinicaltrials.gov registration no.: NCT00702598). A central computerised randomisation process was generated by an independent statistician, stratified for site and conducted in random blocks of 4 or 8 . Concealment of allocation was accomplished using an automated online system that revealed the treatment allocation only after the unique participant number was entered.

\section{Study treatments}

\section{Medication}

Escitalopram was flexibly dosed starting with $10 \mathrm{mg} / \mathrm{day}$ and increasing to $20 \mathrm{mg} /$ day from week 2 at the discretion of the treating physician. 


\section{CBT condition}

We used a telephone-administered CBT programme that is based on a published manual ${ }^{30}$ and validated in an RCT in primary care. $^{31}$ The telephone-CBT programme is modified to be more brief than traditional CBT (30-40 min instead of $60 \mathrm{~min}$ per session) and was offered at convenient times, including evening and weekend sessions. A total of eight telephone CBT sessions were scheduled over 8-10 weeks; the initial session occurred within 2 weeks of randomisation, and subsequent sessions occurred weekly. The initial session focused on motivationenhancement exercises, whereas subsequent sessions emphasised identifying, challenging and distancing from negative thoughts, and the final session focused on a personal care plan and selfmanagement skills. There was no systematic consideration of work-related issues in this programme. The CBT providers were $\mathrm{PhD}$ - or Master's degree-level experienced therapists who received formal training by the developers of the treatment manual ${ }^{30}$ and fidelity was monitored by inspection of therapist task checklists for each session and review of random audiotaped sessions.

\section{Control condition}

A research coordinator provided a 10-minute structured telephone call weekly for 8 weeks, with enquiry about progress and reminders to take medication properly.

\section{Statistical analysis}

Given that the work functioning scales do not have clearly established minimal clinically important differences, for this proof of concept study we determined the sample size using a power analysis calculation based on change in the main clinical outcome (change in MADRS score) within each treatment group, with alpha set to 0.05 and power at $75 \%$. A sample of 50 participants per group was calculated to detect a minimum effect size (Cohen's $d$, standardised difference in mean scores) of 0.5 , regarded as a medium-sized, clinically relevant effect between treatment conditions.

Analysis was conducted based on a modified intent-to-treat (mITT) sample comprising randomised patients who had at least one valid post-randomisation assessment. Missing data were imputed using last observation carried forward (LOCF). An observed-case completer analysis was also conducted on the sample of participants with data at the primary week 12 end-point.

The pre-specified clinical outcome was the adjusted mean change from baseline to end-point (12 weeks) in the MADRS score using LOCF (mITT). All comparisons were analysed using ANCOVA, adjusting for baseline value and site. The planned secondary outcome analyses were conducted similarly, when appropriate. Categorical data (such as proportions of participants with adverse events, response and remission rates) were analysed using chi-squared tests or Fisher's exact test where cell sizes warrant. Because the secondary outcomes (both clinical and work functioning) were considered exploratory analyses, we did not statistically correct for multiple comparisons. Results are presented as means (s.d.).

\section{Results}

Figure 1 shows the flow of patients in the protocol. There were 105 participants initially randomised, with 99 evaluable participants in the mITT analysis ( $n=48$ allocated to the telephone CBT group and $n=51$ to the escitalopram-alone group). There were $40(83 \%)$ completers evaluated at the 12-week end-point for the telephoneCBT group and $46(90 \%)$ for the escitalopram-alone group.
Table 1 shows the baseline and demographic information on the mITT sample. There were no significant baseline differences between the groups in age, gender or other demographic variables.

\section{Clinical outcomes}

The mean dose of escitalopram in the study was $16.0 \mathrm{mg} /$ day (s.d.=4.7); 36\% of participants were taking $10 \mathrm{mg} / \mathrm{day}, 7 \%$ were taking $15 \mathrm{mg} /$ day, and $58 \%$ were taking $20 \mathrm{mg} /$ day. There were no significant differences in escitalopram dose between the telephone-CBT and escitalopram-alone groups $(15.3 \mathrm{mg} /$ day (s.d. =4.7) v. $16.7 \mathrm{mg} /$ day (s.d. $=4.7)$ respectively; $P=$ n.s.). The mean number of CBT sessions completed by the participants was 6.4 (s.d.=2.8), indicating good adherence to the telephoneCBT programme. Therapist fidelity to the CBT programme was also very good; averaged across all sessions, $90 \%$ of all tasks on the therapist checklist were completed (range $83 \%$ to $97 \%$ for each task).

Table 2 shows the summary of clinical outcomes. There were no significant differences in baseline scores between conditions. There was significant improvement in change scores on the MADRS within each treatment condition, with large baselineto-end-point effect sizes ( $d=1.78$ and $d=1.72$ respectively). However, there was no significant difference between the telephone-CBT and escitalopram-alone groups, with only a small observed effect size $(d=0.16)$.

There were also no significant differences in rates of response (defined as $\geqslant 50 \%$ improvement from baseline) or remission

Table 1 Baseline clinical and demographic information of patients in the two treatment conditions $(n=99)$

\begin{tabular}{|c|c|c|}
\hline & $\begin{array}{l}\text { Escitalopram }+ \\
\text { telephone CBT } \\
\qquad(n=48)\end{array}$ & $\begin{array}{l}\text { Escitalopram + } \\
\text { control reminders } \\
\qquad(n=51)\end{array}$ \\
\hline Age, years: mean (s.d.) & $42.3(10.4)$ & $44.2(9.9)$ \\
\hline \multicolumn{3}{|l|}{ Gender, $n(\%)$} \\
\hline Female & $27(56)$ & $27(53)$ \\
\hline Male & $21(44)$ & $24(47)$ \\
\hline \multicolumn{3}{|l|}{ Marital status, $n(\%)$} \\
\hline Married or cohabiting & $20(42)$ & $18(35)$ \\
\hline Separated, divorced, widowed & $11(23)$ & $20(39)$ \\
\hline Never married & $17(35)$ & $13(26)$ \\
\hline \multicolumn{3}{|l|}{ Children, $n$ (\%) } \\
\hline Yes & $25(52)$ & $24(47)$ \\
\hline No & $23(48)$ & $27(53)$ \\
\hline \multicolumn{3}{|l|}{ Education } \\
\hline High school diploma & $5(11)$ & $5(10)$ \\
\hline Some college & $15(32)$ & $16(31)$ \\
\hline College degree & $12(26)$ & $12(24)$ \\
\hline Graduate education & $12(26)$ & $15(29)$ \\
\hline \multicolumn{3}{|l|}{ Job type, $n(\%)$} \\
\hline \multicolumn{3}{|l|}{ Executive, manager } \\
\hline or professional & $19(40)$ & $16(31)$ \\
\hline Sales, clerical, or technician & $17(35)$ & $23(45)$ \\
\hline Production, labourer or service & $12(25)$ & $12(24)$ \\
\hline \multicolumn{3}{|l|}{ Income, $n(\%)$} \\
\hline 0-CAN $\$ 40000$ & $17(35)$ & $20(39)$ \\
\hline CAN\$40 000-CAN\$80 000 & $26(54)$ & $26(51)$ \\
\hline$>$ CAN\$80000 & $5(10)$ & $5(10)$ \\
\hline \multicolumn{3}{|l|}{$\begin{array}{l}\text { Major depressive disorder } \\
\text { recurrence, } n(\%)\end{array}$} \\
\hline Single episode & $13(27)$ & $20(39)$ \\
\hline Recurrent & $35(73)$ & $31(61)$ \\
\hline Number of episodes, mean (s.d.) & $3.9(4.9)$ & $4.0(8.0)$ \\
\hline $\begin{array}{l}\text { Length of current episode, days: } \\
\text { mean (s.d.) }\end{array}$ & $156.4(310.2)$ & $152.8(318.0)$ \\
\hline
\end{tabular}




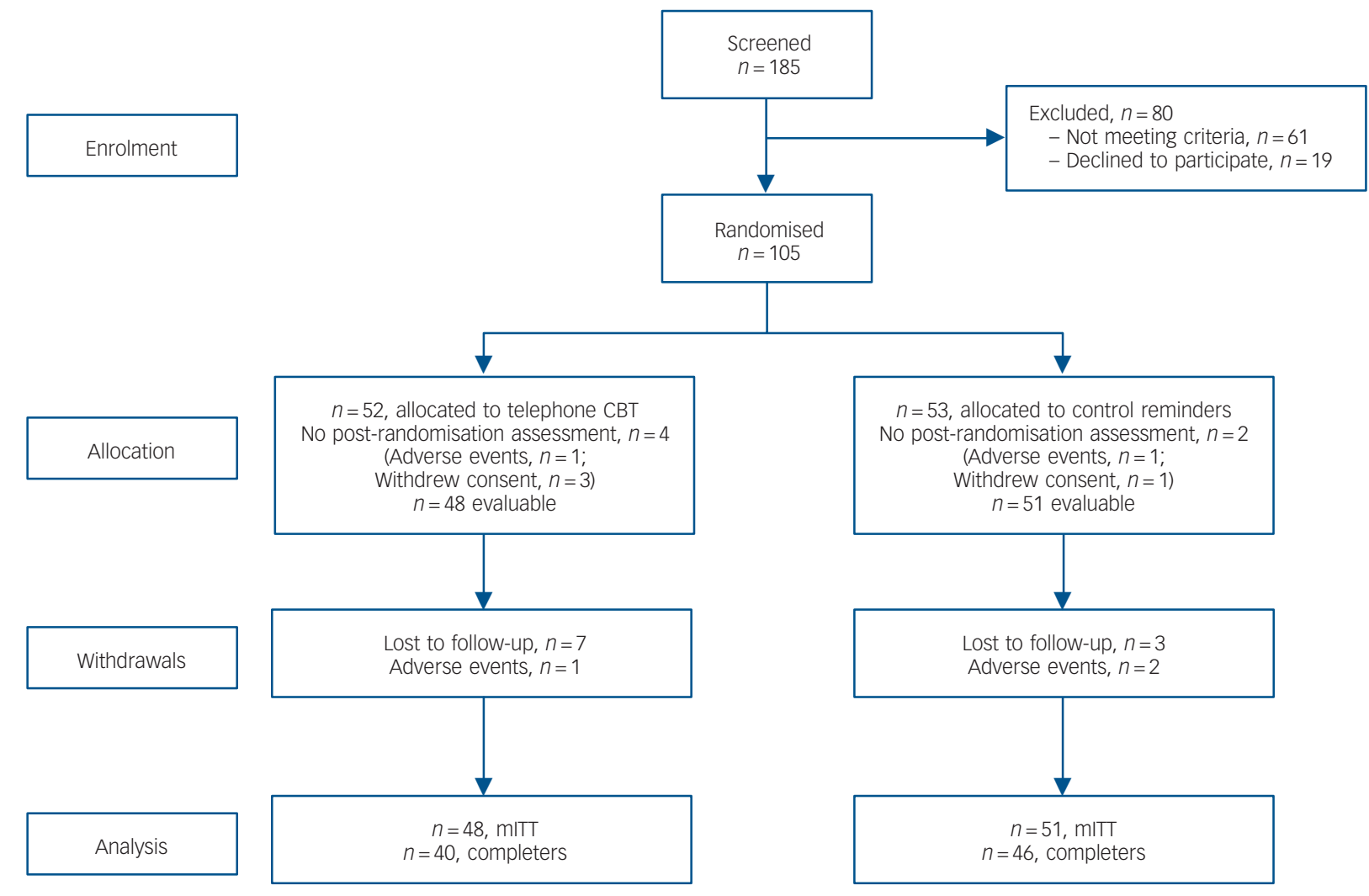

Fig. 1 Flow chart of participants in the protocol. CBT, cognitive-behavioural therapy; mITT, modified intent-to-treat.

(defined as MADRS $\leqslant 12$ ). In the LOCF analysis, response rates were $63 \%$ in the telephone-CBT group and $61 \%$ in the escitalopram-alone group $\left(\chi^{2}=0.03\right.$, d.f. $\left.=1, P=0.86\right)$; remission rates were $56 \%$ v. $53 \%$ respectively $\left(\chi^{2}=0.11\right.$, d.f. $\left.=1, P=0.74\right)$. The rates were also similar in the observed-case completer analyses (those participants who completed the 12 -week evaluation) between the telephone-CBT and escitalopram-alone groups (response: $73 \%$ v. $63 \%, \chi^{2}=0.87$, d.f. $=1, P=0.35$; remission: $65 \%$ v. $54 \%, \chi^{2}=1.00$, d.f. $\left.=1, P=0.38\right)$.

\section{Work functioning outcomes}

Table 3 shows the work functioning outcomes for the two treatment conditions. There were no significant baseline differences between conditions for any outcome. The SDSWork/Role item showed improvement within each treatment condition, but there were no significant differences between treatment conditions in the change from baseline to end-point in the LOCF or completer analyses. The effect sizes were considered small ( $d=0.20$ and $d=0.29$ respectively).

The other work functioning scales did show significant differences between treatment conditions. In the LOCF analysis, the telephone-CBT group had a significantly greater improvement than the escitalopram-alone group on the LEAPS total score $(9.7$ (s.d. = 5.8) v. $6.9($ s.d. $=5.4) ; F=4.07$, d.f. $=1,95, P=0.046)$ and on the LEAPS productivity subscale $(3.9($ s.d. $=2.5) v .2 .6($ s.d. $=2.5)$; $F=4.52$, d.f. $=1,95, P=0.036$ ). Similar results were found in the completer analyses, with effect sizes (ranging from $d=0.46$ to 0.51 ) in the small to medium range. There was also a significant difference in the HPQ Overall Performance change scores. The telephone-CBT group showed significantly greater improvement than the escitalopram-alone condition, with an effect size of 0.48 in the LOCF analysis. Although the effect size $(d=0.47)$ was similar in the completer analysis, the difference in change scores did not reach statistical significance.

\section{Table 2 Clinical outcomes at baseline and at 12-week end-point}

\begin{tabular}{|c|c|c|c|c|c|c|c|c|c|c|}
\hline \multirow[b]{2}{*}{ Clinical outcome time point } & \multicolumn{3}{|c|}{ Escitalopram + telephone CBT } & \multicolumn{3}{|c|}{ Escitalopram + control reminders } & \multicolumn{4}{|c|}{ Analysis } \\
\hline & $n$ & Mean & s.d. & $n$ & Mean & s.d. & $F$ & d.f. & $P$ & $\begin{array}{l}\text { Effect size } \\
\text { (Cohen's d) }\end{array}$ \\
\hline \multicolumn{11}{|l|}{$\begin{array}{l}\text { Montgomery-Åsberg } \\
\text { Depression Rating Scale }\end{array}$} \\
\hline Baseline & 48 & 28.2 & 5.1 & 51 & 27.1 & 4.9 & & & & \\
\hline End-point (LOCF) & 48 & 12.5 & 9.1 & 51 & 12.8 & 8.4 & & & & \\
\hline Change (LOCF) & 48 & 15.7 & 8.8 & 51 & 14.3 & 8.3 & 0.28 & 1,95 & 0.60 & 0.16 \\
\hline End-point (completer) & 40 & 10.3 & 7.6 & 46 & 12.4 & 8.1 & & & & \\
\hline Change (completer) & 40 & 17.3 & 8.3 & 46 & 14.8 & 8.1 & 1.86 & 1,82 & 0.18 & 0.29 \\
\hline
\end{tabular}




\begin{tabular}{|c|c|c|c|c|c|c|c|c|c|c|}
\hline \multirow[b]{2}{*}{ Work outcome time point } & \multicolumn{3}{|c|}{ Escitalopram + telephone CBT } & \multicolumn{3}{|c|}{ Escitalopram + control reminders } & \multicolumn{4}{|c|}{ Analysis } \\
\hline & $n$ & Mean & s.d. & $n$ & Mean & s.d. & $F$ & d.f. & $P$ & $\begin{array}{l}\text { Effect size } \\
\text { (Cohen's d) }\end{array}$ \\
\hline \multicolumn{11}{|l|}{$\begin{array}{l}\text { Sheehan Disability Scale - } \\
\text { Work/Role item }\end{array}$} \\
\hline Baseline & 48 & 6.3 & 2.0 & 51 & 5.8 & 2.6 & & & & \\
\hline End-point (LOCF) & 48 & 3.3 & 2.3 & 51 & 3.3 & 2.9 & & & & \\
\hline Change (LOCF) & 48 & 3.0 & 2.7 & 51 & 2.5 & 2.9 & 0.24 & 1,95 & 0.63 & 0.20 \\
\hline End-point (completer) & 39 & 2.9 & 2.0 & 45 & 3.0 & 2.8 & & & & \\
\hline Change (completer) & 39 & 3.4 & 2.6 & 45 & 2.6 & 2.9 & 0.37 & 1,80 & 0.55 & 0.29 \\
\hline \multicolumn{11}{|c|}{$\begin{array}{l}\text { Lam Employment Absence and } \\
\text { Productivity Scale - total score }\end{array}$} \\
\hline Baseline & 48 & 16.3 & 4.6 & 51 & 14.9 & 5.1 & & & & \\
\hline End-point (LOCF) & 48 & 6.6 & 4.6 & 51 & 8.0 & 5.2 & & & & \\
\hline Change (LOCF) & 48 & 9.7 & 5.8 & 51 & 6.9 & 5.4 & 4.07 & 1,95 & 0.046 & 0.49 \\
\hline End-point (completer) & 37 & 6.7 & 3.8 & 45 & 7.4 & 4.7 & & & & \\
\hline change (completer) & 37 & 10.0 & 5.8 & 45 & 7.4 & 5.1 & 4.35 & 1,78 & 0.035 & 0.47 \\
\hline \multicolumn{11}{|c|}{$\begin{array}{l}\text { Lam Employment Absence and } \\
\text { Productivity Scale - Productivity } \\
\text { subscale }\end{array}$} \\
\hline Baseline & 48 & 6.1 & 2.2 & 51 & 5.4 & 2.6 & & & & \\
\hline End-point (LOCF) & 48 & 2.2 & 1.7 & 51 & 2.8 & 2.2 & & & & \\
\hline Change (LOCF) & 48 & 3.9 & 2.5 & 51 & 2.6 & 2.5 & 4.52 & 1,95 & 0.036 & 0.51 \\
\hline End-point (completer) & 37 & 2.5 & 1.7 & 49 & 2.8 & 2.2 & & & & \\
\hline Change (completer) & 37 & 3.9 & 2.6 & 49 & 2.7 & 2.6 & 4.35 & 1,82 & 0.044 & 0.46 \\
\hline \multicolumn{11}{|c|}{$\begin{array}{l}\text { Health and Work Performance } \\
\text { Questionnaire - overall } \\
\text { performance }\end{array}$} \\
\hline Baseline & 48 & 5.2 & 1.6 & 51 & 5.8 & 2.0 & & & & \\
\hline End-point (LOCF) & 48 & 6.4 & 1.9 & 51 & 6.3 & 1.8 & & & & \\
\hline Change (LOCF) & 48 & -1.2 & 1.5 & 51 & -0.45 & 1.6 & 4.00 & 1,95 & 0.048 & 0.48 \\
\hline End-point (completer) & 36 & 6.4 & 1.7 & 45 & 6.3 & 1.9 & & & & \\
\hline Change (completer) & 36 & -1.3 & 1.6 & 45 & -0.53 & 1.7 & 2.26 & 1,77 & 0.14 & 0.47 \\
\hline
\end{tabular}

Work absence was calculated from questions on the LEAPS asking about hours of work scheduled and hours of work missed over the previous 2-week period. From baseline to end-point, the escitalopram-alone group had numerically higher reduction in hours of work missed than the telephone-CBT group in both LOCF and completer analyses, but these differences were not statistically significant (Table 4). Similarly, there were numerical advantages that were not statistically significant for the escitalopram-alone group in the percentages of work-hours missed/work-hours scheduled.

\section{Adverse events}

There was one serious adverse event in the study in the escitalopram-alone group - an accidental fatality that was

\begin{tabular}{|c|c|c|c|c|c|c|c|c|c|c|}
\hline \multirow[b]{2}{*}{ Work outcome time point } & \multicolumn{3}{|c|}{ Escitalopram + telephone CBT } & \multicolumn{3}{|c|}{ Escitalopram + control reminders } & \multicolumn{4}{|c|}{ Analysis } \\
\hline & $n$ & Mean & s.d. & $n$ & Mean & s.d. & $F$ & d.f. & $P$ & $\begin{array}{l}\text { Effect size } \\
\text { (Cohen's } d \text { ) }\end{array}$ \\
\hline \multicolumn{11}{|l|}{$\begin{array}{l}\text { Hours of work missed } \\
\text { (in past } 2 \text { weeks) }\end{array}$} \\
\hline Baseline & 48 & 9.3 & 16.1 & 51 & 9.5 & 12.6 & & & & \\
\hline End-point (LOCF) & 48 & 7.1 & 11.9 & 51 & 6.7 & 15.4 & & & & \\
\hline Change (LOCF) & 48 & 2.3 & 10.8 & 51 & 2.8 & 15.9 & 0.02 & 1,95 & 0.90 & -0.04 \\
\hline End-point (completer) & 37 & 5.4 & 8.3 & 45 & 6.5 & 15.9 & & & & \\
\hline Change (completer) & 37 & 0.9 & 11.1 & 45 & 2.9 & 16.9 & 0.003 & 1,78 & 0.96 & -0.14 \\
\hline \multicolumn{11}{|c|}{$\begin{array}{l}\text { Hours of work missed/work hours } \\
\text { scheduled (in past } 2 \text { weeks), \% }\end{array}$} \\
\hline Baseline & 48 & 14.1 & 23.3 & 51 & 15.7 & 18.7 & & & & \\
\hline End-point (LOCF) & 48 & 11.5 & 20.9 & 51 & 9.4 & 19.6 & & & & \\
\hline Change (LOCF) & 48 & 2.5 & 20.9 & 51 & 6.2 & 21.0 & 0.23 & 1,95 & 0.64 & -0.18 \\
\hline End-point (completer) & 34 & 10.0 & 19.3 & 45 & 9.0 & 20.2 & & & & \\
\hline Change (completer) & 34 & 0.72 & 23.7 & 45 & 6.5 & 22.3 & 0.37 & 1,75 & 0.54 & -0.25 \\
\hline
\end{tabular}


unrelated to the study and occurred after the primary 12 -week end-point. There were five participants who withdrew early because of adverse events (Fig. 1): two within the first 2 weeks (insomnia $n=1$, agitation/insomnia $n=1$ ), and three in the $3 \mathrm{rd}$ to 8th week of treatment (sexual dysfunction $n=1$, haemorrhoids $n=1$, agitation $n=1)$. The adverse events occurring in more than $10 \%$ of participants were nausea $(n=33,33 \%)$, headache $(n=17$, $17 \%)$, dry mouth $(n=14,14 \%)$, dizziness $(n=12,12 \%)$ and sleepiness $(n=11,11 \%)$. Most adverse events were rated as mild, occurred early in treatment and resolved within the first 4 weeks. There were no significant differences in adverse events between treatment conditions.

\section{Acceptability of telephone СВT}

In a satisfaction survey completed by participants $(n=39)$ in the telephone-CBT group at completion of the study, $13(33 \%)$ participants indicated that they were satisfied and $18(46 \%)$ that they were highly satisfied with the therapy. In addition, 35 (89\%) participants would recommend telephone CBT to a friend or relative with depression.

\section{Discussion}

To our knowledge, this is the first RCT to focus on occupational and productivity outcomes in employed patients with major depressive disorder following treatment with combined psychotherapy and pharmacotherapy. Previously, a pooled analysis of ten clinical trials using individual patient data examined work functioning using a subscale of the Social Adjustment Scale ${ }^{32}$ in a subset of patients with depression treated with tricyclic antidepressants and monoamine oxidase inhibitors and psychotherapy, but there was no separate analysis of the few combined psychotherapy and pharmacotherapy conditions. ${ }^{33}$

Previous RCTs of monotherapy and combination treatments in major depressive disorder have not been optimally designed to examine productivity outcomes. For example, study samples usually included a mix of unemployed and employed patients, or those on short- and long-term disability, making work productivity difficult to assess. Employed people are often unable to participate in clinical trials because of the time-intensive research assessments requiring long study visits for interviews and completion of questionnaires. Further, studies of psychotherapy require patients to take additional time off work to attend therapy appointments. Finally, few treatment studies in major depressive disorder have included specific measures of work functioning as outcomes. Our study methodology addressed these issues by: enrolling only employed patients; minimising in-person study visit frequency and duration by using telephone-based assessments and online questionnaires; conducting CBT via telephone; and using validated scales to assess work functioning. Using this methodology the participants minimised the time away from work, since the telephone and online assessments and the telephone CBT could be scheduled at convenient times, such as during lunch breaks, evenings and weekends. Hence, the methods used in this study are less likely than traditional RCTs to confound and/or interfere with work absence or productivity.

The main results showed that adjunctive telephoneadministered CBT in combination with escitalopram did not result in differences in symptom-based clinical outcomes compared with escitalopram alone; however, the combination treatment did result in superior improvement in some aspects of work productivity, as assessed by instruments designed to assess productivity change. Telephone-administered psychotherapy has been shown in meta-analyses to be more effective than treatment-as-usual conditions in reducing symptoms of depression ${ }^{34}$ but there are no RCTs of combined treatment with antidepressants. Escitalopram is an effective antidepressant with some evidence for superiority over other antidepressants. ${ }^{35,36}$ The large pre-post MADRS effect size $(d=1.7)$ and high rates of response and remission in the escitalopram-alone group may have rendered it difficult to show additional clinical benefit of combination treatment.

\section{Work functioning and major depressive disorder}

There are few studies of antidepressants in employed patients with major depressive disorder that have specifically examined work functioning. Dunlop et $a l^{37}$ evaluated desvenlafaxine $v$. placebo in 429 gainfully employed (working $\geqslant 20 \mathrm{~h} /$ week) patients with major depressive disorder, using the SDS and the Work Productivity and Activity Impairment scale (WPAI). ${ }^{38}$ They found significant superiority of desvenlafaxine in improvement on the SDS-Work/Role item compared with placebo, but differences on the WPAI narrowly missed significance $(P=0.054)$.

The telephone-CBT group in our study showed statistically significant small to medium effect sizes in improvement on several validated, self-rated scales of work productivity: the LEAPS total score $(d=0.49)$ and productivity subscale score $(d=0.51)$, and the HPQ Overall Performance score $(d=0.48)$. Although it must be noted that, as for most functional measures, minimal clinically important differences and clinically meaningful differences have not yet been defined for productivity and impairment scales, these differences are likely clinically important. As an example, the 2.8-point difference in LEAPS total score between conditions represents $41 \%$ of the pre-post improvement in LEAPS score in the escitalopram-alone group.

In contrast to the results in the work functioning scales, there were no differences between treatment conditions in the number of work-hours missed or the percentage of work-hours missed/ work-hours scheduled. These were calculated based on the previous 2 -week period and it is possible that this is not an adequate time period to assess for absences. However, absenteeism may also be problematic as an outcome because it is often dependent on external factors such as corporate sick leave policies, the availability of other staff to cover the work, and the type and nature of the job. Hence, work absence may not be a sensitive measure of change within a short-term clinical trial.

In this study, telephone CBT was well accepted by patients, with $79 \%$ of participants rating themselves as satisfied or highly satisfied with the therapy. However, the drop-out rate not related to medication was numerically (but not statistically) higher in the telephone-CBT group $(n=10,19 \%)$ than the escitalopram-alone group $(n=4,8 \%)$. It was also higher than the reported attrition rate of $7.6 \%$ from the meta-analysis of telephone-delivered psychotherapy, ${ }^{34}$ but comparable to the attrition rate of telephone psychotherapy $(21 \%)$ in an $\mathrm{RCT}^{19}$ in which face-to-face psychotherapy had significantly higher attrition rates (33\%). These results indicate that the telephone is an acceptable delivery method for CBT.

The contrasting results on the MADRS and the work functioning scales in this study are consistent with those of other clinical trials of major depressive disorder showing discrepant results between symptom-based and functioning outcomes. For example, in a 24-week RCT comparing escitalopram with duloxetine, there were no differences in MADRS change scores, response or remission rates, but the escitalopram condition had greater improvement on SDS total and Work/Role item scores. ${ }^{39}$ Furthermore, systematic reviews have shown highly variable correlations between symptom-based and functional outcome measures in studies of major depressive disorder. ${ }^{40}$ Our results 
are consistent with these findings and add support to the recommendation that treatment studies of major depressive disorder should include both symptom and functional scales to assess outcomes. ${ }^{40-42}$

\section{Limitations}

Some limitations of this study must be considered. First, the sample size of this study may have limited power to detect smaller effect sizes in clinical and functional outcomes. We also did not control for multiple statistical comparisons. Second, work functioning and productivity was measured only with self-rating scales. There are few studies using objective measures of productivity, but there are methodological challenges in objectively assessing productivity in many occupations. However, some studies have shown that self-rated work functioning scales show good correlation with objective assessments of work productivity. ${ }^{43}$ Third, the participants were not masked to treatment condition and the control condition consisted of adherence reminder telephone calls that were more brief than the telephone CBT sessions, so we cannot exclude the possibility that attention, patient expectations or other non-specific factors led to superior work outcomes with telephone CBT. Fourth, the telephone-delivered CBT used in this study was a brief (eight 30-minute sessions) intervention designed for use in primary care settings. It is possible that a more standard, longer course of CBT (e.g. 12-16 sessions) or modifying the CBT to focus on work situations, may lead to greater changes in both symptoms and work functioning.

In summary, this proof of concept study found that combining telephone-administered CBT with a first-line antidepressant may improve self-reported work functioning in employed patients with major depressive disorder compared with an antidepressant alone. Given the importance of work-related outcomes to patients and to society, it will be important to assess work absence and productivity outcomes in more treatment studies of major depressive disorder, in order to optimally reduce depression-related occupational impairment and the burden of presenteeism.

\footnotetext{
Raymond W. Lam, MD, Department of Psychiatry, University of British Columbia, UBC Hospital, Vancouver, British Columbia; Sagar V. Parikh, MD, Department of Psychiatry, University of Toronto, University Health Network, Toronto, Ontario; Psychiatry, University of Toronto, University Health Network, Toronto, Ontario;
Rajamannar Ramasubbu, MD, Department of Psychiatry, University of Calgary, Calgary, Alberta; Erin E. Michalak, PhD, Edwin M. Tam, MD, Auby Axler, MD, Lakshmi N. Yatham, MBBS, Department of Psychiatry, University of British Columbia, UBC Hospital, Vancouver, British Columbia; Sidney H. Kennedy, MD, Department of Psychiatry, University of Toronto, University Health Network, Toronto, Ontario; Chinnapalli v. Manjunath, MD, Department of Psychiatry, University of British Columbia, Surrey Memorial Hospital, Surrey, British Columbia, Canada

Correspondence: Dr Raymond W. Lam, Department of Psychiatry, University of British Columbia, 2255 Wesbrook Mall, Vancouver, BC, V6T 2A1, Canada. Email: r.lam@ubc.ca

First received 17 Dec 2012, final revision 16 Apr 2013, accepted 5 Jun 2013
}

\section{Funding}

This study was funded by an investigator-initiated unrestricted grant from Lundbeck Canada. The sponsor had no role in the design and conduct of the study, in the collection, analysis, and interpretation of the data, or in the preparation or approval of the manuscript.

\section{Acknowledgements}

The authors thank Cindy Woo and Tanya Poitras for their work in coordinating this trial.

\section{References}

1 Myette L, Garuso G, Stave G. Position Statement: Depression in the Working Population. American College of Occupational and Environmental Medicine, 2009
2 Bender A, Farvolden P. Depression and the workplace: a progress report. Curr Psychiatry Rep 2008; 10: 73-9.

3 Kessler RC, Merikangas KR, Wang PS. The prevalence and correlates of workplace depression in the national comorbidity survey replication. J Occup Environ Med 2008; 50: 381-90.

4 Veronese A, Ayuso-Mateos JL, Cabello M, Chatterji S, Nuevo R. Work disability and depressive disorders: impact on the European population. Am J Phys Med Rehabil 2012; 91 (13 suppl 1): S62-8.

5 LaMontagne AD, Sanderson K, Cocker F. Estimating the Economic Benefits of Eliminating Job Strain as a Risk Factor for Depression. Victorian Heath Promotion Foundation, 2010.

6 World Health Organization. The Global Burden of Disease: 2004 Update. WHO, 2008.

7 Towers Watson. The Health and Productivity Advantage: 2009/2010 Staying@Work Report. Towers Watson, 2010.

8 Aronsson G, Gustafsson K. Sickness presenteeism: prevalence, attendancepressure factors, and an outline of a model for research. J Occup Environ Med 2005; 47: 958-66.

9 Collins JJ, Baase CM, Sharda CE, Ozminkowski RJ, Nicholson S, Billotti GM, et al. The assessment of chronic health conditions on work performance, absence, and total economic impact for employers. J Occup Environ Med 2005; 47: 547-57.

10 Lerner D, Henke RM. What does research tell us about depression, job performance, and work productivity? J Occup Environ Med 2008; 50: 401-10.

11 Beck A, Crain AL, Solberg LI, Unutzer J, Glasgow RE, Maciosek MV, et al. Severity of depression and magnitude of productivity loss. Ann Fam Med 2011; 9: 305-11.

12 Goetzel RZ, Long SR, Ozminkowski RJ, Hawkins K, Wang S, Lynch W. Health, absence, disability, and presenteeism cost estimates of certain physical and mental health conditions affecting U.S. employers. J Occup Environ Med 2004; 46: 398-412.

13 Birnbaum HG, Ben-Hamadi R, Kelley D, Hsieh M, Seal B, Kantor E, et al. Assessing the relationship between compliance with antidepressant therapy and employer costs among employees in the United States. J Occup Environ Med 2010; 52: 115-24.

14 Luo Z, Cowell AJ, Musuda YJ, Novak SP, Johnson EO. Course of major depressive disorder and labor market outcome disruption. J Ment Health Policy Econ 2010; 13: 135-49.

15 Dewa CS, Thompson $\mathrm{AH}$, Jacobs $\mathrm{P}$. The association of treatment of depressive episodes and work productivity. Can J Psychiatry 2011; 56: $743-50$.

16 Nieuwenhuijsen $\mathrm{K}$, Bultmann $\mathrm{U}$, Neumeyer-Gromen A, Verhoeven AC, Verbeek JH, Feltz-Cornelis $\mathrm{CM}$. Interventions to improve occupational health in depressed people. Cochrane Database Syst Rev 2008; 2: CD006237.

17 Kennedy SH, Lam RW, Parikh SV, Patten SB, Ravindran AV. Canadian Network for Mood and Anxiety Treatments (CANMAT) clinical guidelines for the management of major depressive disorder in adults. Introduction. J Affect Disord 2009; 117 (suppl 1): S1-2.

18 Bee $\mathrm{PE}$, Bower $\mathrm{P}$, Lovell $\mathrm{K}$, Gilbody S, Richards $\mathrm{D}$, Feltz-Cornelis CM Psychotherapy mediated by remote communication technologies: a meta-analytic review. BMC Psychiatry 2008; 8: 60.

19 Mohr DC, Ho J, Deffecy J, Reifler D, Sokol L, Burns MN, et al. Effect of telephone-administered vs face-to-face cognitive behavioral therapy on adherence to therapy and depression outcomes among primary care patients. A randomized trial. JAMA 2012; 307: 2278-85.

20 Simon GE, Ludman EJ, Tutty S, Operskalski B, Von Korff M. Telephone psychotherapy and telephone care management for primary care patients starting antidepressant treatment: a randomized controlled trial. JAMA 2004; 292: 935-42.

21 Wang PS, Simon GE, Avorn J, Azocar F, Ludman EJ, McCulloch J, et al. Telephone screening, outreach, and care management for depressed workers and impact on clinical and work productivity outcomes: a randomized controlled trial. JAMA 2007; 298: 1401-11.

22 Parikh SV, Segal ZV, Grigoriadis S, Ravindran AV, Kennedy SH, Lam RW, et al. Canadian Network for Mood and Anxiety Treatments (CANMAT) clinical guidelines for the management of major depressive disorder in adults. II. Psychotherapy alone and in combination with antidepressant medications. J Affect Disord 2009; 117 (suppl 1): S15-25.

23 Sheehan DV, Lecrubier $Y$, Sheehan $\mathrm{KH}$, Amorim $\mathrm{P}$, Janavs J, Weiller $\mathrm{E}$, et al. The Mini-International Neuropsychiatric Interview (M.I.N.I.): the development and validation of a structured diagnostic psychiatric interview for DSM-IV and ICD-10. J Clin Psychiatry 1998; 59 (suppl 20): 22-33.

24 Cuijpers P, Dekker J, Hollon SD, Andersson G. Adding psychotherapy to pharmacotherapy in the treatment of depressive disorders in adults: a meta-analysis. J Clin Psychiatry 2009; 70: 1219-29. 
25 de Maat SM, Dekker J, Schoevers RA, de Jonghe F. Relative efficacy of psychotherapy and combined therapy in the treatment of depression: a meta-analysis. Eur Psychiatry 2007; 22: 1-8.

26 Montgomery SA, Åsberg M. A new depression scale designed to be sensitive to change. Br J Psychiatry 1979; 134: 382-9.

27 Leon AC, Olfson M, Portera L, Farber L, Sheehan DV. Assessing psychiatric impairment in primary care using the Sheehan Disability Scale. Int J Psychiatry Med 1997; 27: 93-105.

28 Lam RW, Michalak EE, Yatham LN. A new clinical rating scale for work absence and productivity: validation in patients with major depressive disorder. BMC Psychiatry 2009; 9: 78.

29 Kessler RC, Barber C, Beck A, Berglund P, Cleary PD, McKenas D, et al. The World Health Organization Health and Work Performance Questionnaire (HPQ). J Occup Environ Med 2003; 45: 156-74.

30 Simon GE, Ludman EJ, Tutty S. Creating a Balance: A Step-By-Step Approach to Managing Stress and Lifting Your Mood. Trafford Publishing, 2006.

31 Tutty S, Ludman EJ, Simon G. Feasibility and acceptability of a telephone psychotherapy program for depressed adults treated in primary care. Gen Hosp Psychiatry 2005; 27: 400-10.

32 Weissman MM, Bothwell S. Assessment of social adjustment by patient self-report. Arch Gen Psychiatry 1976; 33: 1111-5.

33 Mintz J, Mintz LI, Arruda MJ, Hwang SS. Treatments of depression and the functional capacity to work. Arch Gen Psychiatry 1992; 49: 761-8.

34 Mohr DC, Vella L, Hart S, Heckman T, Simon G. The effect of telephoneadministered psychotherapy on symptoms of depression and attrition: a meta-analysis. Clin Psychol (New York) 2008; 15: 243-53.

35 Cipriani A, Furukawa TA, Salanti G, Geddes JR, Higgins JP, Churchill R, et al. Comparative efficacy and acceptability of 12 new-generation antidepressants: a multiple-treatments meta-analysis. Lancet 2009; 373 : 746-58.

36 Ali MK, Lam RW. Comparative efficacy of escitalopram in the treatment of major depressive disorder. Neuropsychiatr Dis Treat 2011; 7: 39-49.

37 Dunlop BW, Reddy S, Yang L, Lubaczewski S, Focht K, Guico-Pabia CJ. Symptomatic and functional improvement in employed depressed patients: a double-blind clinical trial of desvenlafaxine versus placebo. J Clin Psychopharmacol 2011; 31: 569-76.

38 Reilly MC, zbrozek AS, Dukes EM. The validity and reproducibility of a work productivity and activity impairment instrument. Pharmacoeconomics 1993: 4: $353-65$.

39 Wade A, Gembert K, Florea I. A comparative study of the efficacy of acute and continuation treatment with escitalopram versus duloxetine in patients with major depressive disorder. Curr Med Res Opin 2007; 23: 1605-14.

40 McKnight PE, Kashdan TB. The importance of functional impairment to mental health outcomes: a case for reassessing our goals in depression treatment research. Clin Psychol Rev 2009; 29: 243-59.

41 Greer TL, Kurian BT, Trivedi MH. Defining and measuring functional recovery from depression. CNS Drugs 2010; 24: 267-84.

42 Lam RW, Filteau MJ, Milev R. Clinical effectiveness: the importance of psychosocial functioning outcomes. J Affect Disord 2011; 132 (suppl 1): S9-13.

43 Kessler RC, Ames M, Hymel PA, Loeppke R, McKenas DK, Richling DE, et al. Using the World Health Organization Health and Work Performance Questionnaire (HPQ) to evaluate the indirect workplace costs of illness. J Occup Environ Med 2004; 46 (6 suppl): S23-37. 